Portland State University

PDXScholar

$9-1-1991$

Field-Equation Approximations and Amplification in High-Gain Lasers: Analytical Results

Lee W. Casperson

Portland State University

Follow this and additional works at: https://pdxscholar.library.pdx.edu/ece_fac

Part of the Electrical and Computer Engineering Commons

Let us know how access to this document benefits you.

Citation Details

Casperson, L. W. (1991). Field-equation approximations and amplification in high-gain lasers: Analytical results. 44 (5), 3305-3316.

This Article is brought to you for free and open access. It has been accepted for inclusion in Electrical and Computer Engineering Faculty Publications and Presentations by an authorized administrator of PDXScholar. Please contact us if we can make this document more accessible: pdxscholar@pdx.edu. 


\title{
Field-equation approximations and amplification in high-gain lasers: Analytical results
}

\author{
Lee W. Casperson* \\ Department of Physics, University of Otago, P. O. Box 56, Dunedin, New Zealand
}

(Received 24 January 1991)

\begin{abstract}
In a related study the equations governing a high-gain laser amplifier have been solved numerically without making the usual slowly-varying-amplitude derivative approximation in Maxwell's equations, and thus the field amplitudes are not restricted to vary negligibly in a distance of one wavelength. The results reveal an instability that involves reflections and oscillatory growth of perturbations away from steady state. In the present study steady-state solutions of the field equations are obtained analytically, and transformations are described for converting the equations to alternative intensity-equation sets. These intensity equations are linearized and solved for the growth rate and oscillation period of the perturbations.
\end{abstract}

\section{INTRODUCTION}

Most models of laser amplifiers incorporate first-order differential equations for the electric field or equivalently first-order rate equations for the intensity or photon density. However, the derivation of these equations from Maxwell's equations requires the elimination of a spatial derivative, and this simplification may not be justified for all laser amplifiers. In a companion study, a theoretical model for laser amplification has been developed that incorporates Maxwell's equations without approximation [1]. Numerical solutions of that model have been obtained, and those solutions reveal reflection and oscillatory instability of a propagating light signal.

The purpose of this study is to explore analytically the same amplifier model that was developed and investigated numerically in Ref. [1]. The model can be written in several different forms, each of which may have advantages for certain types of investigation. The original model in which the field variables appear explicitly is relatively difficult to work with, but any solutions to that model contain the most complete information about the propagating waves. Much simpler equation sets are also possible involving quadratic functions of the field variables, such as intensities and energy densities, as the dependent variables. These intensity equations contain no information about the phases of the field components, but they lead more directly to the other quantities of practical interest. Various analytic solutions are obtained for steady-state loss-limited propagation in laser amplifiers and for the complex oscillation frequencies of perturbations away from steady state. The complete $z$ - dependent solutions are reviewed for the simpler models that incorporate the slowly-varying-amplitude derivative approximation in Maxwell's equations.

Steady-state solutions of the general field-equation model are derived in Sec. II. These solutions include explicit formulas for the steady-state field energy and wavelength. In Sec. III it is shown how the model may be reformulated in terms of variables that are quadratic in the field amplitudes, and the most practical of these models involve the intensities and energy densities of the propagating waves. Advantages of these formulations include a reduced number of independent equations and a simpler form of the saturation denominators. In Sec. IV an intensity model is linearized and solved to obtain stability criteria for perturbations away from the steady-state solution. One of the solutions of the resulting eigenvalue equation is a negative real quantity, which corresponds to the same damped behavior that is obtained for the conventional amplifier models that include the slowlyvarying-amplitude derivative approximation in Maxwell's equations. The other two solutions are a complexconjugate pair with positive real part, and these solutions show that steady-state propagation in a laser amplifier is unstable with respect to oscillatory growth of any perturbations.

\section{STEADY-STATE SOLUTIONS}

The formalism on which this study is based was discussed in detail in Ref. [1], and a range of numerical solutions were described. The starting point for this analysis is the non-Doppler inhomogeneously broadened version of the set of equations given in Ref. [1] as Eqs. (80)-( 83):

$$
\begin{aligned}
\frac{d B_{r}(\zeta)}{d \zeta}=- & \left(2(1-f) A_{r}(\xi)+\delta\left[\left(y+z_{0}\right) A_{i}(\xi)-\left(y_{0}+z_{0}\right) B_{i}(\zeta)\right]\right. \\
& \left.+\frac{2\left(y+z_{0}\right)}{z_{0}} \int_{-\infty}^{\infty} \frac{(y-U) A_{i}(\zeta)-A_{r}(\zeta)}{1+(y-U)^{2}+A_{r}^{2}(\zeta)+A_{i}^{2}(\zeta)} D_{0}(U, \zeta) d U\right],
\end{aligned}
$$




$$
\begin{aligned}
& \begin{aligned}
\frac{d B_{i}(\zeta)}{d \zeta}=- & 2(1-f) A_{i}(\zeta)-\delta\left[\left(y+z_{0}\right) A_{r}(\zeta)-\left(y_{0}+z_{0}\right) B_{r}(\zeta)\right]
\end{aligned}\left.\quad-\frac{2\left(y+z_{0}\right)}{z_{0}} \int_{-\infty}^{\infty} \frac{(y-U) A_{r}(\zeta)+A_{i}(\zeta)}{1+(y-U)^{2}+A_{r}^{2}(\zeta)+A_{i}^{2}(\zeta)} D_{0}(U, \zeta) d U\right] \\
& \frac{d A_{r}(\zeta)}{d \zeta}=-\left\{2 f B_{r}(\zeta)+\delta\left[\left(y+z_{0}\right) B_{i}(\zeta)-\left(y_{0}+z_{0}\right) A_{i}(\zeta)\right]\right\} \\
& \frac{d A_{i}(\zeta)}{d \zeta}=-\left\{2 f B_{i}(\zeta)-\delta\left[\left(y+z_{0}\right) B_{r}(\zeta)-\left(y_{0}+z_{0}\right) A_{r}(\zeta)\right]\right\}
\end{aligned}
$$

where $\zeta=\gamma_{c} z / c_{1}$ is a normalized distance with $c_{1}=\left(\mu_{1} \epsilon_{1}\right)^{-1 / 2}$ the background speed of light and $\gamma_{c}=\sigma / 2 \epsilon_{1}$ the electric-field decay rate. The variables $A_{r}(\zeta)$ and $A_{i}(\zeta)$ are, respectively, the real and imaginary parts of the normalized electric field, $B_{r}(\xi)$ and $B_{i}(\xi)$ are the corresponding parts of the normalized magnetic field, $\delta=\gamma / \gamma_{c}$ is a dimensionless decay rate ratio with $\gamma$ the decay rate of the off-diagonal density-matrix elements, $y=\left(\omega-\omega_{0}\right) / \gamma$ is the normalized lasing frequency offset from some frequency $\omega_{0}$ that is characteristic of the transition, $y_{0}=\left(\Omega-\omega_{0}\right) / \gamma$ is the normalized offset of the frequency $\Omega=k\left(\mu_{1} \epsilon_{1}\right)^{-1 / 2}$ that defines the arbitrarily chosen propagation constant $k$ or wavelength $\lambda=2 \pi / k$ in the amplifier, $z_{0}=\omega_{0} / \gamma$ is the normalized characteristic frequency of the transition, $U=\left(\omega_{\alpha}-\omega_{0}\right) / \gamma$ is the normalized frequency offset of the laser transition for members of an atomic or molecular class $\alpha, D_{0}(U, \zeta)$ is the normalized unsaturated population difference distribution for the non-Doppler inhomogeneously broadened medium, and the factor $f$ allows an arbitrary fraction of the losses to be associated with the magnetic field while not affecting the overall normalization.

Equations (1)-(4) reduce to a much simpler model in the limit of $z_{0}$ approaching infinity. It follows from Eqs. (3) and (4) that in this limit the electric- and magneticfield components approach equality. Then if one substitutes Eqs. (3) and (4) into Eqs. (1) and (2) and uses the equalities just mentioned, the magnetic variables can be eliminated, and Eqs. (1) and (2) reduce to the set

$$
\begin{aligned}
\frac{d A_{r}(\zeta)}{d \zeta}=- & A_{r}(\zeta)+\delta\left(y-y_{0}\right) A_{i}(\zeta) \\
& +\int_{-\infty}^{\infty} \frac{(y-U) A_{i}(\zeta)-A_{r}(\zeta)}{1+(y-U)^{2}+A_{r}^{2}(\zeta)+A_{i}^{2}(\zeta)} \\
& \left.\times D_{0}(U, \zeta) d U\right] \\
\frac{d A_{i}(\zeta)}{d \zeta}=- & {\left[A_{i}(\zeta)-\delta\left(y-y_{0}\right) A_{r}(\zeta)\right.} \\
& -\int_{-\infty}^{\infty} \frac{(y-U) A_{r}(\zeta)+A_{i}(\zeta)}{1+(y-U)^{2}+A_{r}^{2}(\zeta)+A_{i}^{2}(\zeta)} \\
& \left.\times D_{0}(U, \zeta) d U\right]
\end{aligned}
$$

These are also the amplifier equations that one obtains by making at the outset the derivative approximation that is being investigated here. Thus a primary purpose of this investigation will be to examine analytically any differences between the predictions of Eqs. (1)-(4) and Eqs. (5) and (6).

One of the most significant differences between the behavior of the two models that have just been summarized is the dramatic spatial instability of the more rigorous model, and this instability was explored numerically in Ref. [1]. It would be especially desirable for practical applications to also obtain analytical criteria for the onset and growth of this instability. The first step toward this goal is to look for any steady-state solutions of the model which may be tested for stability. Steady-state behavior of a laser amplifier corresponds to the loss-limited condition in which the laser gain is exactly balanced by distributed losses. To establish a background for this study, we will first look for steady-state solutions of the approximate model given in Eqs. (5) and (6).

In looking for steady-state solutions of Eqs. (5) and (6) the derivatives will be set to zero, and the field variables and pump rate will be assumed to be independent of the distance $\zeta$. In this limit the equations reduce to

$$
\begin{aligned}
0= & A_{r}+\delta\left(y-y_{0}\right) A_{i} \\
& +\int_{-\infty}^{\infty} \frac{(y-U) A_{i}-A_{r}}{1+(y-U)^{2}+A_{r}^{2}+A_{i}^{2}} D_{0}(U) d U, \\
0= & A_{i}-\delta\left(y-y_{0}\right) A_{r} \\
& -\int_{-\infty}^{\infty} \frac{(y-U) A_{r}+A_{i}}{1+(y-U)^{2}+A_{r}^{2}+A_{i}^{2}} D_{0}(U) d U .
\end{aligned}
$$

An obvious solution of these equations is the zero-field case $A_{r}=A_{i}=0$. The more interesting loss-limited case is obtained from a simultaneous solution of the nonlinear equations.

To obtain nonzero solutions of Eqs. (7) and (8) it is helpful to first simplify the notation to

$0=A_{r}+\delta\left(y-y_{0}\right) A_{i}+a\left(y, A_{r}, A_{i}\right) A_{i}-b\left(y, A_{r}, A_{i}\right) A_{r}$,

$$
0=A_{i}-\delta\left(y-y_{0}\right) A_{r}-a\left(y, A_{r}, A_{i}\right) A_{r}-b\left(y, A_{r}, A_{i}\right) A_{i},
$$


where the new inhomogeneous integral functions are defined by

$a\left(y, A_{r}, A_{i}\right)=\int_{-\infty}^{\infty} \frac{y-U}{1+(y-U)^{2}+A_{r}^{2}+A_{i}^{2}} D_{0}(U) d U$, $b\left(y, A_{r}, A_{i}\right)=\int_{-\infty}^{\infty} \frac{1}{1+(y-U)^{2}+A_{r}^{2}+A_{i}^{2}} D_{0}(U) d U$

Nontrivial solutions of Eqs. (9) and (10) are only possible if the determinant of the coefficients of the field variables is zero:

$$
\left|\begin{array}{cc}
1-b\left(y, A_{r}, A_{i}\right) & \delta\left(y-y_{0}\right)+a\left(y, A_{r}, A_{i}\right) \\
-\delta\left(y-y_{0}\right)-a\left(y, A_{r}, A_{i}\right) & 1-b\left(y, A_{r}, A_{i}\right)
\end{array}\right|=0 .
$$

Expanding the determinant yields the equation

$$
\left[1-b\left(y, A_{r}, A_{i}\right)\right]^{2}+\left[\delta\left(y-y_{0}\right)+a\left(y, A_{r}, A_{i}\right)\right]^{2}=0 .
$$

The squared terms in this equation must individually equal zero, and one obtains the two conditions

$$
\begin{aligned}
& b\left(y, A_{r}, A_{i}\right)=1, \\
& a\left(y, A_{r}, A_{i}\right)=-\delta\left(y-y_{0}\right) .
\end{aligned}
$$

With Eq. (12) it is clear that Eq. (15) is basically an equation determining the electric energy density of the nontrivial loss-limited solution, and this energy density will be defined here as $U_{e}=A_{r}^{2}+A_{i}^{2}$ [1]. The meaning of the electric energy density will be discussed further in Sec. III. With this quantity determined, Eqs. (11) and (16) can be interpreted as determining the value of the parameter $y_{0}$ according to

$$
y_{0}=y+a\left(y, A_{r}, A_{i}\right) / \delta \text {. }
$$

With the definition of $y_{0}$ given above, it is clear that Eq. (17) establishes the value of the wavelength $\lambda$ such that the phase of the fields is constant. If the inhomogeneous population difference function $D_{0}(U)$ were a Gaussian, for example, the energy density and wavelength would be obtained from implicit equations involving error functions. For brevity only the limit of homogeneous broadening will be considered in detail.

For a homogeneously broadened laser amplifier, the population difference function $D_{0}(U)$ may be replaced by a $\delta$ function in $U$ times the constant population difference $D_{0}$. This parameter $D_{0}$ may also be replaced by the threshold parameter $r$, which is the ratio of the pumping rate to its value when the unsaturated gain at line center is just sufficient to make the field derivatives vanish [1]. With these substitutions Eqs. (11) and (12) reduce to

$$
\begin{aligned}
& a\left(y, A_{r}, A_{i}\right)=\frac{y r}{1+y^{2}+U_{e}}, \\
& b\left(y, A_{r}, A_{i}\right)=\frac{r}{1+y^{2}+U_{e}} .
\end{aligned}
$$

With Eq. (15) it follows from Eq. (19) that the electric energy density is

$$
U_{e}=r-1-y^{2}
$$

With Eqs. (17), (18), and (20), the parameter $y_{0}$ can be written

$$
\begin{aligned}
y_{0} & =y+\frac{1}{\delta} \frac{y r}{1+y^{2}+U_{e}} \\
& =y\left(1+\delta^{-1}\right) .
\end{aligned}
$$

Equation (21) shows, in effect, that the steady-state wavelength differs from its background value $\lambda=2 \pi c_{1} / \omega$ if $\delta$ is not large compared to unity.

Equations (20) and (21) give the solutions for the steady-state energy density and wavelength in a losslimited laser amplifier where the usual approximation of small gain per wavelength has been employed. The same basic techniques are also applicable to the more rigorous laser model given in Eqs. (1)-(4). For steady-state operation these equations become

$$
\begin{aligned}
0= & 2(1-f) A_{r}+\delta\left[\left(y+z_{0}\right) A_{i}-\left(y_{0}+z_{0}\right) B_{i}\right] \\
& +2\left(y+z_{0}\right) a A_{i} / z_{0}-2\left(y+z_{0}\right) b A_{r} / z_{0}, \\
0= & 2(1-f) A_{i}-\delta\left[\left(y+z_{0}\right) A_{r}-\left(y_{0}+z_{0}\right) B_{r}\right] \\
& -2\left(y+z_{0}\right) a A_{r} / z_{0}-2\left(y+z_{0}\right) b A_{i} / z_{0}, \\
0= & 2 f B_{r}+\delta\left[\left(y+z_{0}\right) B_{i}-\left(y_{0}+z_{0}\right) A_{i}\right], \\
0= & 2 f B_{i}-\delta\left[\left(y+z_{0}\right) B_{r}-\left(y_{0}+z_{0}\right) A_{r}\right],
\end{aligned}
$$

where the integral notation introduced in Eqs. (11) and (12) has again been employed, and for brevity the dependent variables in the integral functions have not been shown. Like the simplified set discussed above, these equations also have the obvious solution in which all of the field components are equal to zero. Nontrivial solutions of Eqs. (22) - (25) are only possible when the determinant of the coefficients of the field variables is equal to zero: 


$$
\left|\begin{array}{cccc}
2(1-f)-2\left(y+z_{0}\right) b / z_{0} & \left(y+z_{0}\right)\left(\delta+2 a / z_{0}\right) & 0 & -\delta\left(y_{0}+z_{0}\right) \\
-\left(y+z_{0}\right)\left(\delta+2 a / z_{0}\right) & 2(1-f)-2\left(y+z_{0}\right) b / z_{0} & \delta\left(y_{0}+z_{0}\right) & 0 \\
0 & -\delta\left(y_{0}+z_{0}\right) & 2 f & \delta\left(y+z_{0}\right) \\
\delta\left(y_{0}+z_{0}\right) & 0 & -\delta\left(y+z_{0}\right) & 2 f
\end{array}\right|=0 .
$$

Expanding the determinant yields the equation

$$
\begin{aligned}
& {\left[\delta^{2}\left(y+z_{0}\right)^{2}+4 f^{2}\right]\left[2(1-f)-2\left(y+z_{0}\right) b / z_{0}\right]^{2}+4 f \delta^{2}\left(y_{0}+z_{0}\right)^{2}\left[2(1-f)-2\left(y+z_{0}\right) b / z_{0}\right]} \\
& \quad+\left[\delta^{2}\left(y+z_{0}\right)^{2}+4 f^{2}\right]\left[\left(y+z_{0}\right)\left(\delta+2 a / z_{0}\right)\right]^{2}-2 \delta^{3}\left(y+z_{0}\right)\left(y_{0}+z_{0}\right)^{2}\left[\left(y+z_{0}\right)\left(\delta+2 a / z_{0}\right)\right]+\delta^{4}\left(y_{0}+z_{0}\right)^{4}=0 .
\end{aligned}
$$

Equation (27) may be written as the sum of two squares:

$$
\begin{aligned}
\left\{\left[\delta^{2}\left(y+z_{0}\right)^{2}+4 f^{2}\right]\left[2(1-f)-2\left(y+z_{0}\right) b / z_{0}\right]+2 f \delta^{2}\left(y_{0}+z_{0}\right)^{2}\right\}^{2} & \\
& +\left\{\left[\delta^{2}\left(y+z_{0}\right)^{2}+4 f^{2}\right]\left[\left(y+z_{0}\right)\left(\delta+2 a / z_{0}\right)\right]-\delta^{3}\left(y+z_{0}\right)\left(y_{0}+z_{0}\right)^{2}\right\}^{2}=0 .
\end{aligned}
$$

Again, the squared terms must individually vanish, and one obtains the conditions

$$
\begin{aligned}
{\left[\delta^{2}\left(y+z_{0}\right)^{2}+4 f^{2}\right][2(1-f)-} & \left.2\left(y+z_{0}\right) b / z_{0}\right] \\
& =-2 f \delta^{2}\left(y_{0}+z_{0}\right)^{2}, \\
{\left[\delta^{2}\left(y+z_{0}\right)^{2}+4 f^{2}\right]\left[\left(y+z_{0}\right)\right.} & \left.\left(\delta+2 a / z_{0}\right)\right] \\
= & \delta^{3}\left(y+z_{0}\right)\left(y_{0}+z_{0}\right)^{2} .
\end{aligned}
$$

These equations are conditions determining the electric energy density $U_{e}$ and the normalized frequency parameter $y_{0}$. In the limit of large $z_{0}$ they reduce, respectively, to the approximate results given in Eqs. (15) and (16). The parameter $y_{0}$ may be eliminated by dividing the two equations, and the result is

$$
\frac{2(1-f)-2\left(y+z_{0}\right) b / z_{0}}{\left(y+z_{0}\right)\left(\delta+2 a / z_{0}\right)}=-\frac{2 f}{\delta\left(y+z_{0}\right)} .
$$

Cross-multiplying and simplifying, this reduces to

$$
\delta\left(y+z_{0}\right) b=\delta z_{0}+2 f a .
$$

For a homogeneously broadened laser, the functions $a\left(y, A_{r}, A_{i}\right)$ and $b\left(y, A_{r}, A_{i}\right)$ are given in Eqs. (18) and (19), and Eq. (32) can be solved for the electric energy density:

$$
U_{e}=\frac{\left[\delta\left(y+z_{0}\right)-2 f y\right] r}{\delta z_{0}}-1-y^{2}
$$

It is clear that in the limit of large $z_{0}$ this exact result reduces to the approximation given above in Eq. (20). It is interesting to notice that agreement with the approximation is also obtained at line center $(y=0)$. In general, however, the exact energy density may be either larger or smaller than the approximate value. For large detunings $\left(y>z_{0}\right)$ and large pump rates $(r>>1)$ the discrepancy between the exact and approximate formulas may be substantial.

After the energy density is known, the parameter $y_{0}$ can be determined from either Eq. (29) or (30). The result is

$$
y_{0}=\left[\frac{\left[\delta^{2}\left(y+z_{0}\right)^{2}+4 f^{2}\right]\left[\delta\left(y+z_{0}\right)+2 y(1-f)\right]}{\delta^{2}\left[\delta\left(y+z_{0}\right)-2 f y\right]}\right]^{1 / 2}-z_{0} .
$$

One can show that for large values of $z_{0}$ this result approaches the approximate formula given in Eq. (21). With line center tuning $(y=0)$, the exact and approximate results are not the same. In general, no nontrivial steady-state solution is possible if $y_{0}$ is set equal to zero at the outset.

The absolute phase of the steady-state solution is indeterminate in the absence of any boundary conditions. Therefore any one of the four field components can be specified arbitrarily. For example, the component $A_{i}$ could be set equal to zero, and $A_{r}$ could be determined from the electric energy density given in Eq. (33). Then the magnetic-field components could be readily obtained from the solution of Eqs. (24) and (25).

\section{INTENSITY FORMALISMS}

After the steady-state values of the field components are known, one can begin to address the question of per- 
turbation stability. In principle, the stability of the steady-state amplifier solution can be assessed by linearizing Eqs. (1)-(4) near steady state and then substituting in a complex exponential trial solution. In practice, however, this approach is cumbersome for general values of the amplifier parameters, and it leads to a quartic equation for the complex oscillation frequencies. A somewhat simpler approach is to first transform the field equations to a new set of equations governing intensities and energy densities. Such a new set will be found to contain three equations rather than four, and it can still describe the intensity instability revealed numerically in Ref. [1].

There are various quadratic intensity- and energyrelated quantities that can form the basis of the transformation mentioned above. As a first example, we will adopt the set

$$
\begin{aligned}
& I(\zeta)=A_{r}(\zeta) B_{r}(\zeta)+A_{i}(\zeta) B_{i}(\zeta) \\
& I^{*}(\zeta)=A_{r}(\zeta) B_{i}(\zeta)-A_{i}(\zeta) B_{r}(\zeta) \\
& U_{e}(\zeta)=A_{r}^{2}(\zeta)+A_{i}^{2}(\zeta) \\
& U_{m}(\zeta)=B_{r}^{2}(\zeta)+B_{i}^{2}(\zeta)
\end{aligned}
$$

The intensity $I$ and energy densities $U_{e}$ and $U_{m}$ were defined previously in Eqs. (72)-(74) of Ref. [1], and the conjugate intensity $I^{*}$ will be shown to provide a convenient closure of the equation set. Using Eqs. (1)-(4) and (35) $-(38)$, a set of equations for the intensities and energy densities can be written in the form

$$
\begin{gathered}
\frac{d I(\zeta)}{d \zeta}=-\left[2(1-f) U_{e}(\zeta)+2 f U_{m}(\zeta)-\frac{2\left(y+z_{0}\right) U_{e}(\zeta)}{z_{0}} \int_{-\infty}^{\infty} \frac{1}{1+(y-U)^{2}+U_{e}(\zeta)} D_{0}(U, \zeta) d U\right] \\
\frac{d I^{*}(\zeta)}{d \zeta}=-\left[\delta\left(y+z_{0}\right)\left[U_{m}(\xi)-U_{e}(\zeta)\right]-\frac{2\left(y+z_{0}\right) U_{e}(\zeta)}{z_{0}} \int_{-\infty}^{\infty} \frac{y-U}{1+(y-U)^{2}+U_{e}(\zeta)} D_{0}(U, \zeta) d U\right], \\
\frac{d U_{e}(\zeta)}{d \zeta}=-2\left[2 f I(\zeta)+\delta\left(y+z_{0}\right) I^{*}(\zeta)\right], \\
\frac{d U_{m}(\zeta)}{d \zeta}=-2\left\{\left[2(1-f)-\frac{2\left(y+z_{0}\right)}{z_{0}} \int_{-\infty}^{\infty} \frac{1}{1+(y-U)^{2}+U_{e}(\zeta)} D_{0}(U, \zeta) d U\right] I(\zeta)\right. \\
\left.\left.-\left(y+z_{0}\right)\left[\delta+\frac{2}{z_{0}}\right] \int_{-\infty}^{\infty} \frac{y-U}{1+(y-U)^{2}+U_{e}(\zeta)} D_{0}(U, \zeta) d U\right] I^{*}(\zeta)\right\}
\end{gathered}
$$

While these new equations are by definition consistent with Eqs. (1)-(4), the two sets are not equivalent. In particular, information about the phase of the propagating wave has been lost in Eqs. (39)-(42), and it is significant that the wavelength determining parameter $y_{0}$ has now dropped out of the model.

For some purposes the disadvantage of losing phase information is more than compensated by the much greater simplicity of the model. One finds, for example, that the four intensity and energy variables defined in Eqs. (35)-(38) are not independent of each other but must satisfy the constraint

$$
U_{e}(\zeta) U_{m}(\zeta)=I^{2}(\zeta)+I^{* 2}(\zeta) .
$$

Therefore any one of the equations in the set of Eqs. (39)-(42) may be regarded as redundant and may be eliminated. If Eq. (43) is used to eliminate the variable $U_{m}(\xi)$, for example, the remaining equations are

$$
\begin{aligned}
& \frac{d I(\zeta)}{d \zeta}=-\left[2(1-f) U_{e}(\zeta)+2 f \frac{I^{2}(\zeta)+I^{* 2}(\zeta)}{U_{e}(\zeta)}-\frac{2\left(y+z_{0}\right) U_{e}(\zeta)}{z_{0}} \int_{-\infty}^{\infty} \frac{1}{1+(y-U)^{2}+U_{e}(\xi)} D_{0}(U, \zeta) d U\right] \\
& \frac{d I^{*}(\zeta)}{d \zeta}=-\left[\delta\left(y+z_{0}\right) \frac{I^{2}(\zeta)+I^{* 2}(\zeta)-U_{e}^{2}(\xi)}{U_{e}(\zeta)}-\frac{2\left(y+z_{0}\right) U_{e}(\xi)}{z_{0}} \int_{-\infty}^{\infty} \frac{y-U}{1+(y-U)^{2}+U_{e}(\zeta)} D_{0}(U, \zeta) d U\right] \\
& \frac{d U_{e}(\zeta)}{d \zeta}=-2\left[2 f I(\zeta)+\delta\left(y+z_{0}\right) I^{*}(\zeta)\right] .
\end{aligned}
$$

Thus the $\zeta$ dependence of the intensity in an amplifier can be obtained by solving only three equations instead of four as done in Ref. [1].

It is useful to consider again the limit $z_{0}=\infty$, in which the standard derivative approximation becomes exact. In this limit Eqs. (44)-(46) reduce to a single familiar equation for the $z$ dependence of the intensity in a laser amplifier. Details of this reduction and a summary of the resulting analytic solutions is included in the Appendix. As a further check on Eqs. (44)-(46) and for later use, 
we consider again the problem of loss-limited steady-state propagation in a laser amplifier. In this limit the equations can be written

$0=2(1-f) U_{e}^{2}+2 f\left(I^{2}+I^{* 2}\right)-\frac{2\left(y+z_{0}\right) U_{e}^{2}}{z_{0}} b\left(y, U_{e}\right)$,

$0=\delta\left(y+z_{0}\right)\left(I^{2}+I^{* 2}-U_{e}^{2}\right)-\frac{2\left(y+z_{0}\right) U_{e}^{2}}{z_{0}} a\left(y, U_{e}\right)$,

$0=2 f I+\delta\left(y+z_{0}\right) I^{*}$,

where the coefficients $a\left(y, U_{e}\right)$ and $b\left(y, U_{e}\right)$ are updated from Eqs. (11) and (12). Equations (47) and (48) may each be solved for the quantity $\left(I^{2}+I^{* 2}\right) / U_{e}^{2}$, and the results are, respectively,

$$
\begin{aligned}
& \frac{I^{2}+I^{* 2}}{U_{e}^{2}}=\frac{y+z_{0}}{f z_{0}} b\left(y, U_{e}\right)-\frac{1-f}{f}, \\
& \frac{I^{2}+I^{* 2}}{U_{e}^{2}}=\frac{2}{\delta z_{0}} a\left(y, U_{e}\right)+1 .
\end{aligned}
$$

Equating the right-hand sides of these equations yields again Eq. (32). Thus the steady-state electric energy predicted by this form of the model is the same as the prediction of the original amplitude equations, and for homogeneous broadening the energy density is given by Eq. (33).

Steady-state solutions for the intensity variables can also be readily obtained. From Eq. (49) the conjugate intensity $I^{*}$ is related to the intensity $I$ by

$$
I^{*}=-\frac{2 f}{\delta\left(y+z_{0}\right)} I .
$$

Equations (51) and (52) may be combined to obtain an equation for the intensity in terms of the energy density

$$
I=\left(\frac{1+\left(2 / \delta z_{0}\right) a\left(y, U_{e}\right)}{1+\left[2 f / \delta\left(y+z_{0}\right)\right]^{2}}\right)^{1 / 2} U_{e} .
$$

For a homogeneously broadened laser the function $a\left(y, U_{e}\right)$ is given by Eq. (18), and the energy density $U_{e}$ is given by Eq. (33). With these substitutions Eq. (53) becomes

$$
\begin{aligned}
I= & {\left[\frac{1+2 y /\left[\delta\left(y+z_{0}\right)-2 f y\right]}{1+\left[2 f / \delta\left(y+z_{0}\right)\right]^{2}}\right]^{1 / 2}, } \\
& \times\left[\frac{\left[\delta\left(y+z_{0}\right)-2 f y\right] r}{\delta z_{0}}-1-y^{2}\right],
\end{aligned}
$$

and Eq. (52) is

$$
\begin{aligned}
I^{*}=- & {\left[\frac{1+2 y /\left[\delta\left(y+z_{0}\right)-2 f y\right]}{1+\left[\delta\left(y+z_{0}\right) / 2 f\right]^{2}}\right]^{1 / 2} } \\
& \times\left[\frac{\left[\delta\left(y+z_{0}\right)-2 f y\right] r}{\delta z_{0}}-1-y^{2}\right] .
\end{aligned}
$$

For some purposes it is useful to decompose the intensity into plus and minus intensity components. As shown in Ref. [1], a useful definition of these components is

$$
\begin{aligned}
& I^{+}(\zeta)=\frac{1}{4}\left[A_{r}(\zeta)+B_{r}(\zeta)\right]^{2}+\frac{1}{4}\left[A_{i}(\zeta)+B_{i}(\zeta)\right]^{2}, \\
& I^{-}(\zeta)=\frac{1}{4}\left[A_{r}(\zeta)-B_{r}(\zeta)\right]^{2}+\frac{1}{4}\left[A_{i}(\zeta)-B_{i}(\zeta)\right]^{2} .
\end{aligned}
$$

Fortunately, it is not necessary to know the individual field components in order to determine the plus and minus intensities. From a comparison of the definitions given in Eqs. (35), (37), (38), (56), and (57), one finds that the plus and minus intensities can be determined from the quantities predicted by Eqs. (43)-(46) by means of the relationships

$$
\begin{aligned}
& I^{+}(\xi)=\frac{U_{e}(\xi)+U_{m}(\xi)}{4}+\frac{I(\xi)}{2}, \\
& I^{-}(\xi)=\frac{U_{e}(\zeta)+U_{m}(\xi)}{4}-\frac{I(\zeta)}{2} .
\end{aligned}
$$

Thus the reduced amplifier model may be used to determine all intensity and energy related aspects of the propagating waves in a medium with arbitrarily high gain or loss.

It is perhaps appropriate to note that other quadratic transformations can also be used to reduce the field equations. One can, for example, use the definitions in Eqs. (56) and (57) together with the new definitions

$$
\begin{aligned}
& u(\zeta)=\frac{1}{4}\left[A_{r}^{2}(\zeta)+A_{i}^{2}(\zeta)-B_{r}^{2}(\zeta)-B_{i}^{2}(\zeta)\right] \\
& u^{*}(\xi)=\frac{1}{2}\left[B_{r}(\zeta) A_{i}(\zeta)-A_{r}(\zeta) B_{i}(\zeta)\right]
\end{aligned}
$$

In terms of these functions, Eqs. (1)-(4) may be used to derive the new set

$$
\begin{aligned}
& \frac{d I^{*}(\zeta)}{d \zeta}=-2\left[I^{+}(\zeta)+(1-2 f) u(\zeta)+\frac{y+z_{0}}{z_{0}} \int_{-\infty}^{\infty} \frac{(y-U) u^{*}(\zeta)-I^{+}(\xi)-u(\xi)}{1+(y-U)^{2}+I^{+}(\zeta)+I^{-}(\xi)+2 u(\xi)} D_{0}(U, \zeta) d U\right], \\
& \frac{d I^{-}(\zeta)}{d \zeta}=+2\left[I^{-}(\zeta)+(1-2 f) u(\xi)-\frac{y+z_{0}}{z_{0}} \int_{-\infty}^{\infty} \frac{(y-U) u^{*}(\xi)+I^{-}(\zeta)+u(\zeta)}{1+(y-U)^{2}+I^{+}(\zeta)+I^{-}(\zeta)+2 u(\zeta)} D_{0}(U, \zeta) d U\right],
\end{aligned}
$$




$$
\begin{aligned}
& \frac{d u(\zeta)}{d \zeta}=-\left[(1-2 f)\left[I^{-}(\zeta)-I^{+}(\zeta)\right]-2 \delta\left(y+z_{0}\right) u^{*}(\zeta)\right. \\
& \left.-\frac{y+z_{0}}{z_{0}} \int_{-\infty}^{\infty} \frac{2(y-U) u^{*}(\zeta)+I^{-}(\xi)-I^{+}(\xi)}{1+(y-U)^{2}+I^{+}(\zeta)+I^{-}(\zeta)+2 u(\xi)} D_{0}(U, \zeta) d U\right], \\
& \frac{d u^{*}(\zeta)}{d \zeta}=-\left[2 \delta\left(y+z_{0}\right) u(\zeta)+\frac{y+z_{0}}{z_{0}} \int_{-\infty}^{\infty} \frac{(y-U)\left[I^{+}(\zeta)+I^{-}(\zeta)+2 u(\zeta)\right]}{1+(y-U)^{2}+I^{+}(\xi)+I^{-}(\zeta)+2 u(\xi)} D_{0}(U, \zeta) d U\right] \text {. }
\end{aligned}
$$

As with the set given above in Eqs. (39) - (42) the phaserelated parameter $y_{0}$ does not appear in these equations. Also, it may be noted that these equations are not all independent. Thus one finds from Eqs. (56), (57), (60), and (61) that the dependent variables are related by

$$
I^{+}(\zeta) I^{-}(\zeta)=u^{2}(\xi)+u^{* 2}(\zeta)
$$

While Eqs. (62)-(66) provide an alternative basis for any calculation concerning energy density or intensity, these equations and other possible sets have no obvious computational advantages over the set given above in Eqs. (43)-(46). That previous set is generally easier to work with; and, with $U_{e}(\xi)$ as a variable, it has the simplest possible form of the saturation denominators. However, as noted in the Appendix, the set given in Eqs. (62)-(66) can provide a connection between the basic rate equation treatments of two-directional laser amplifiers and the more exact models emphasized here.

\section{STABILITY CRITERIA}

In Ref. [1] it was shown by means of numerical solutions that the propagation of electromagnetic waves in high-gain amplifying media may be unstable. This instability leads, for example, to oscillatory growth of perturbations of the steady-state loss-limited intensity and, viewed differently, to reflection of a propagating wave. The purpose of this section is to derive analytic expressions for the instability behavior for certain illustrative special cases. The starting point for this analysis is the homogeneous limit of Eqs. (44)-(46):

$$
\begin{aligned}
& \frac{d I(\zeta)}{d \zeta}=-\left(2(1-f) U_{e}(\xi)+2 f \frac{I^{2}(\zeta)+I^{* 2}(\zeta)}{U_{e}(\zeta)}\right. \\
& \left.-\frac{2\left(y+z_{0}\right) U_{e}(\zeta)}{z_{0}} \frac{1}{1+y^{2}+U_{e}(\zeta)} D_{0}(\zeta)\right], \\
& \frac{d I^{*}(\zeta)}{d \zeta}=-\left[\delta\left(y+z_{0}\right) \frac{I^{2}(\zeta)+I^{* 2}(\zeta)-U_{e}^{2}(\zeta)}{U_{e}(\zeta)}\right. \\
& \left.-\frac{2\left(y+z_{0}\right) U_{e}(\zeta)}{z_{0}} \frac{y}{1+y^{2}+U_{e}(\xi)} D_{0}(\zeta)\right]
\end{aligned}
$$

$$
\frac{d U_{e}(\zeta)}{d \zeta}=-2\left[2 f I(\zeta)+\delta\left(y+z_{0}\right) I^{*}(\zeta)\right] .
$$

In the simplest case all of the losses are associated with the electric fields, and the parameter $f$ may be set equal to zero. Then, for a uniformly pumped amplifier Eqs. $(67)-(69)$ reduce to

$$
\begin{aligned}
& \frac{d I(\zeta)}{d \zeta}=-2 U_{e}(\zeta)\left(1-\frac{y+z_{0}}{z_{0}} \frac{r}{1+y^{2}+U_{e}(\zeta)}\right), \\
& \frac{d I^{*}(\zeta)}{d \zeta}=-\left[\delta\left(y+z_{0}\right) \frac{I^{2}(\zeta)+I^{* 2}(\zeta)-U_{e}^{2}(\zeta)}{U_{e}(\zeta)}\right. \\
& \left.-\frac{2\left(y+z_{0}\right) U_{e}(\zeta)}{z_{0}} \frac{y r}{1+y^{2}+U_{e}(\zeta)}\right), \\
& \frac{d U_{e}(\zeta)}{d \zeta}=-2 \delta\left(y+z_{0}\right) I^{*}(\zeta) \text {. }
\end{aligned}
$$

The stability of the loss-limited intensity can be evaluated by examining the behavior of small perturbations away from the steady-state solutions. From Eqs. (33), (54), and (55), the steady-state solutions of Eqs. (70)-(72) are

$$
\begin{aligned}
& I=\left[1+\frac{2 y}{\delta\left(y+z_{0}\right)}\right]^{1 / 2}\left[\frac{\left(y+z_{0}\right)}{z_{0}} r-1-y^{2}\right], \\
& I^{*}=0 \\
& U_{e}=\frac{\left(y+z_{0}\right)}{z_{0}} r-1-y^{2} .
\end{aligned}
$$

The $\zeta$-dependent perturbations away from steady-state are defined by the equations

$$
\begin{aligned}
& I(\zeta)=I+I^{\prime}(\zeta) \\
& I^{*}(\zeta)=I^{*}+I^{* \prime}(\zeta) \\
& U_{e}(\zeta)=U_{e}+U_{e}^{\prime}(\zeta)
\end{aligned}
$$

When Eqs. (76) - (78) are substituted into Eqs. (70)-(72) and the results linearized, one obtains the linear equation set

$$
\frac{d I^{\prime}(\zeta)}{d \zeta}=-\frac{2 z_{0}}{\left(y+z_{0}\right) r}\left(\frac{\left(y+z_{0}\right)}{z_{0}} r-1-y^{2}\right) U_{e}^{\prime}(\zeta),
$$




$$
\begin{aligned}
\frac{d I^{* \prime}(\zeta)}{d \zeta}= & -2 \delta\left(y+z_{0}\right)\left[1+\frac{2 y}{\delta\left(y+z_{0}\right)}\right)^{1 / 2} I^{\prime} \\
& +\left[2 \delta\left(y+z_{0}\right)\right. \\
& \left.+2 y\left[1+\frac{z_{0}}{\left(y+z_{0}\right) r}\left(1+y^{2}\right)\right]\right] U_{e}^{\prime}(\zeta),
\end{aligned}
$$

$\frac{d U_{e}^{\prime}(\zeta)}{d \zeta}=-2 \delta\left(y+z_{0}\right) I^{* \prime}(\zeta)$,

where the steady-state parameter values have been eliminated using Eqs. (73)-( 75 ).

The derivatives in Eqs. (79)-(81) can be eliminated by means of the exponential substitutions

$$
\begin{aligned}
& I^{\prime}(\xi)=I^{\prime \prime} \exp (s \xi) \\
& I^{* \prime}(\zeta)=I^{*}{ }^{\prime \prime} \exp (s \zeta), \\
& U_{e}^{\prime}(\xi)=U_{e}^{\prime \prime} \exp (s \zeta)
\end{aligned}
$$

Thus the linear differential equations reduce to the linear algebraic equations

$$
\begin{aligned}
s I^{\prime \prime}= & -\frac{2 z_{0}}{\left(y+z_{0}\right) r}\left[\frac{\left(y+z_{0}\right)}{z_{0}} r-1-y^{2}\right) U_{e}^{\prime \prime}, \\
s I^{* \prime \prime}= & -2 \delta\left(y+z_{0}\right)\left[1+\frac{2 y}{\delta\left(y+z_{0}\right)}\right]^{1 / 2} I^{\prime \prime} \\
& +\left[2 \delta\left(y+z_{0}\right)+2 y\left[1+\frac{z_{0}}{\left(y+z_{0}\right) r}\left(1+y^{2}\right)\right]\right] U_{e}^{\prime \prime},
\end{aligned}
$$

$s U_{e}^{\prime \prime}=-2 \delta\left(y+z_{0}\right) I^{* \prime \prime}$.

These equations can be combined to obtain a cubic equation for the complex frequencies $s$.

As a final simplification, it is assumed that the laser is tuned to the center frequency of the transition $(y=0)$. Then Eqs. $(85)-(87)$ reduce to

$$
\begin{aligned}
& s I^{\prime \prime}=-\frac{2}{r}(r-1) U_{e}^{\prime \prime}, \\
& s I^{* \prime \prime}=-2 \delta z_{0} I^{\prime \prime}+2 \delta z_{0} U_{e}^{\prime \prime}, \\
& s U_{e}^{\prime \prime}=-2 \delta z_{0} I^{* \prime \prime} .
\end{aligned}
$$

These equations may be combined to obtain the relatively elementary cubic equation

$$
s^{3}+4 \delta^{2} z_{0}^{2} s+8 \delta^{2} z_{0}^{2}(r-1) / r=0 .
$$

With the definition $d=4 \delta^{2} z_{0}^{2}$, this equation can be written in the more compact form

$$
s^{3}+d s+2 d(r-1) / r=0 .
$$

Equation (92) has three roots, and techniques for ex- tracting those roots are well known. In one analytical approach, the roots can be written in the form [2]

$$
\begin{aligned}
& s_{1}=F+G, \\
& s_{2}=-\frac{F+G}{2}+\frac{F-G}{2} 3^{1 / 2} i, \\
& s_{3}=-\frac{F+G}{2}-\frac{F-G}{2} 3^{1 / 2} i,
\end{aligned}
$$

where for the present example the parameters $F$ and $G$ are given by

$F=+\left[-\frac{d(r-1)}{r}+\left[\frac{d^{2}(r-1)^{2}}{r^{2}}+\frac{d^{3}}{27}\right]^{1 / 2}\right]^{1 / 3}$,
$G=-\left[+\frac{d(r-1)}{r}+\left[\frac{d^{2}(r-1)^{2}}{r^{2}}+\frac{d^{3}}{27}\right]^{1 / 2}\right]^{1 / 3}$.

Thus $s_{1}$ is always a negative real number, while $s_{2}$ and $s_{3}$ form a complex-conjugate pair. It is significant already that the conjugate pair always has a positive real part, and hence one-directional propagation in this laser amplifier is always unstable against the type of reflections that have been discussed more fully in Ref. [1]. In view of these results it is convenient to introduce the three length definitions

$$
\begin{aligned}
& \zeta_{d}=-1 /(F+G), \\
& \zeta_{g}=-2 /(F+G), \\
& \zeta_{p}=4 \pi /\left[3^{1 / 2}(F-G)\right],
\end{aligned}
$$

where $\zeta_{d}$ is the damping length of the decaying solution, $\zeta_{g}$ is the gain length of the unstable solutions, and $\zeta_{p}$ is the oscillation period of the unstable solutions.

The lengths $\xi_{d}$ and $\xi_{g}$ are plotted in Fig. 1 as functions of the threshold parameter $r$ for various values of the normalized frequency parameter $d$. It is clear from this figure that $\zeta_{d}$ and $\zeta_{g}$ vary little with $d$, but vary substantially with $r$ for small values of $r$. The oscillation period $\zeta_{p}$ is plotted in Fig. 2 as a function of $r$ for various values of $d$. The period is evidently almost independent of $r$ but decreases steadily for increasing values of $d$. In applying these results to actual laser amplifiers, further simplifications would often be possible.

In some practical lasers the parameter $d$ would be much larger than $27(r-1)^{2} / r^{2}$. If one makes the corresponding simplification of the square-root terms in Eqs. (96) and (97), the results are

$$
\begin{aligned}
& F=+\left[(d / 3)^{3 / 2}-d(r-1) / r\right]^{1 / 3}, \\
& G=-\left[(d / 3)^{3 / 2}+d(r-1) / r\right]^{1 / 3} .
\end{aligned}
$$

A first-order expansion of these remaining terms yields

$$
\begin{aligned}
& F=+(d / 3)^{1 / 2}\left[1-(3 / d)^{1 / 2}(r-1) / r\right], \\
& G=-(d / 3)^{1 / 2}\left[1+(3 / d)^{1 / 2}(r-1) / r\right] .
\end{aligned}
$$

In this approximation the roots of the cubic equation given in Eqs. (93) - (95) become

$$
s_{1}=-2(r-1) / r \text {, }
$$




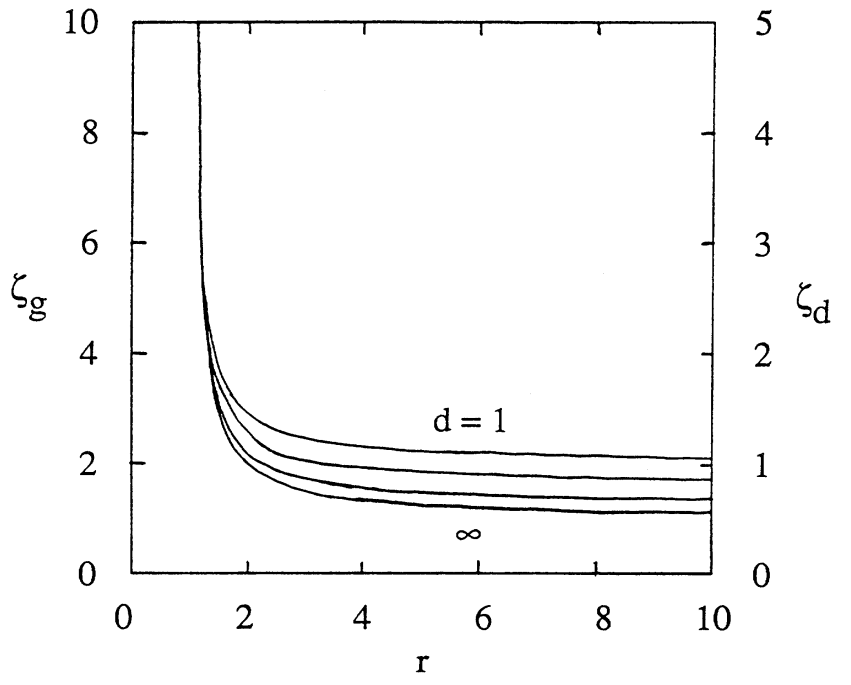

FIG. 1. Damping length $\zeta_{d}$ for the decaying solution and the gain length $\zeta_{g}$ for the oscillatory solution for perturbations of the steady-state loss-limited intensity in a laser amplifier. The length constants are plotted as functions of the threshold parameter $r$ for the frequency parameter values (a) $d=1$, (b) $d=2.5$, (c) $d=10$, and (d) $d=\infty$. The damping lengths vary strongly with $r$ but are somewhat independent of $d$.

$$
\begin{aligned}
& s_{2}=(r-1) / r+d^{1 / 2} i, \\
& s_{3}=(r-1) / r-d^{1 / 2} i,
\end{aligned}
$$

Thus the gain length $\zeta_{g}=r /(r-1)$ is now independent of $d$, and the oscillation period $\zeta_{p}=2 \pi / d^{1 / 2}$ is independent of $r$.

The exponentially decaying solution corresponding to

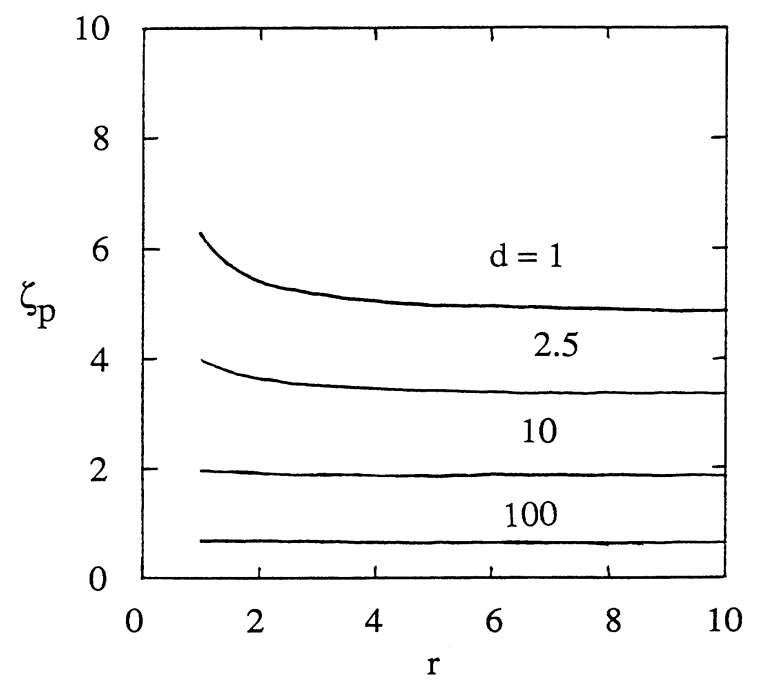

FIG. 2. Oscillation period $\zeta_{p}$ for perturbations of the losslimited solution in a laser amplifier. The period is plotted as a function of the threshold parameter $r$ for the frequency parameter values (a) $d=1$, (b) $d=2.5$, (c) $d=10$, and (d) $d=100$. The oscillation period varies strongly with $d$ but is somewhat independent of $r$. the root $s_{1}$ is not unique to the more exact solutions developed here. It is shown in the Appendix that this root is also obtained for the decay rate of perturbations away from steady state for the familiar approximate homogeneous laser model. On the other hand, the unstable complex-conjugate root pair does not occur in laser models which include the derivative approximation. These roots may, however, be compared with the oscillatory instabilities revealed by the numerical solutions in Ref. [1]. For example, with $r=20$ a perturbation should grow by a factor of $e$ in a normalized distance of about $\xi_{g}=r /(r-1)=1.053$, and this rate is in reasonable agreement with the numerical results shown in Fig. 1 of Ref. [1]. Similarly, with a decay-rate ratio of $\delta=0.3$ and various normalized frequencies $z_{0}$, the predicted oscillation length $\zeta_{p}=\pi / \delta z_{0}$ is in good agreement with the numerically computed oscillations shown in Ref. [1].

Other approximate forms of the complex oscillation roots may also be of interest. In most practical laser amplifiers the unsaturated gain is much larger than the distributed losses, and hence the parameter $r$ is much larger than unity. Thus Eqs. (96) and (97) could usually be simplified to

$$
\begin{aligned}
& F=+\left[-d+d(1+d / 27)^{1 / 2}\right]^{1 / 3}, \\
& G=-\left[+d+d(1+d / 27)^{1 / 2}\right]^{1 / 3} .
\end{aligned}
$$

Using these formulas in Eqs. (93)-(95), the various damping and gain lengths and oscillation periods are plotted in Fig. 3. For large values of both $r$ and $d$ the roots given in Eqs. (93)-(95) are simply

$$
\begin{aligned}
& s_{1}=-2, \\
& s_{2}=1+d^{1 / 2} i, \\
& s_{3}=1-d^{1 / 2} i .
\end{aligned}
$$

The calculations described here have emphasized the

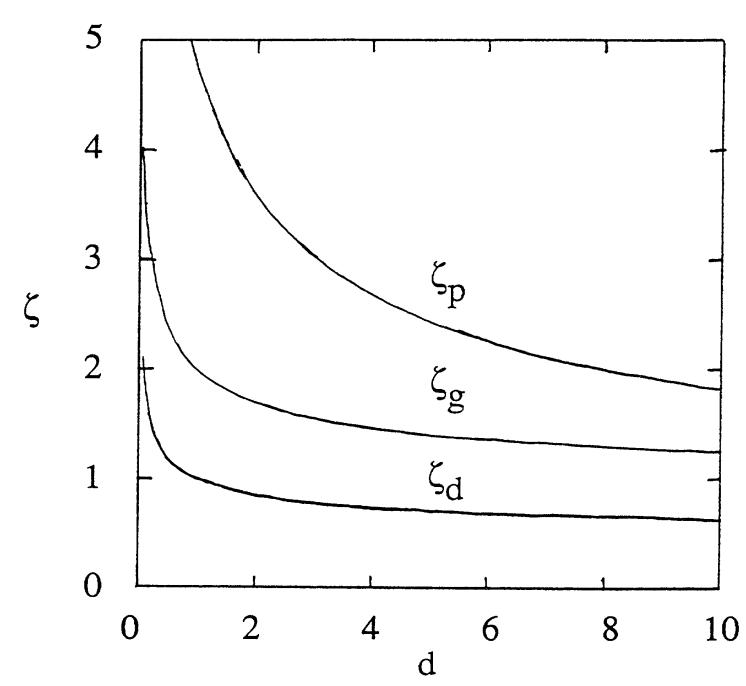

FIG. 3. Damping length $\zeta_{d}$, gain length $\zeta_{g}$, and oscillation period $\zeta_{p}$ as functions of the frequency parameter $d$ for large values of the threshold parameter $r$. 
simplest case of homogeneous line broadening. It is also possible to carry out a stability analysis of laser amplifiers in the inhomogeneous limit using the method introduced in Ref. [3]. However, the resulting eigenvalue equations would be much more complicated than for the homogeneous limit.

\section{CONCLUSION}

In Ref. [1] numerical amplifier models have been developed which avoid the usual approximation in the field equations that the field amplitudes vary negligibly in a wavelength. The improved models revealed striking instability and reflection effects that are not present in the simpler models. In the present study several analytic transformations and simplifications of the amplifier models have been explored. It has been shown that the basic four-equation model for the field amplitudes can be replaced by simpler three equation sets for the intensities and energy densities, and in appropriate limits these sets reduce to the conventional equations for one-directional and two-directional laser amplifiers. Stability analyses can also be carried out analytically, and as an illustration the stability of the one-directional amplifier equations has been examined in detail. It is found that one-directional amplification may be unstable, and the growth of small perturbations of a propagating steady-state loss-limited signal can also be interpreted in terms of growth of a backward-propagating wave.

It is of interest to see whether the effects described here might have significance for practical laser amplifiers, and the analytical results make such an evaluation straightforward. First of all, one may enquire as to the expected size of the relevant parameters in the model. The frequency parameter may be written $d=4 \delta^{2} z_{0}^{2}=4 \omega_{0}^{2} / \gamma_{c}^{2}$ and thus is a quantity on the order of the squared reciprocal of the fractional energy loss per optical cycle. In most laser amplifiers the loss per cycle would be small, so $d$ would be a large number. It follows from Eqs. (106) and (107) that for large values of $d$ the growth constant for the instability is $\zeta_{g}=r /(r-1)$ and thus is a number on the order of unity. But the parameter $\zeta_{g}$ is normalized to the decay length associated with background losses, and in many lasers this length is comparable to the overall amplifier length. In dye lasers, for example, there may be substantial absorption of the laser signal due to other nonlasing transitions; and in semiconductor diode lasers band-to-band absorption is sometimes not much less than the laser gain. In such lasers significant reflections and substantial growth of any perturbations should occur within a single pass, and experiments to demonstrate some of the effects described here should be straightforward.

\section{ACKNOWLEDGMENTS}

This work was supported in part by the National Science Foundation and by Tektronix, Inc. The author also expresses his appreciation to W. J. Sandle, R. J. Ballagh, D. M. Warrington, N. J. Mulgan, and other members of the Department of Physics at the University of Otago for valuable discussions and hospitality during his sabbatical visit.

\section{APPENDIX: APPROXIMATE AMPLIFIER MODELS}

In the preceding text an analytic model has been developed for the propagation of the intensities and energy densities in a laser amplifier. In one formulation an intensity variable corresponding to one-directional propagation appears explicitly, while in a closely related formulation two intensity variables appear representing the plus and minus waves in a two-directional amplifier. We have not obtained general analytic solutions for these models, but it is useful to see how they reduce to known results for the limits corresponding to the usual derivative approximation.

The three-equation form of the amplifier model structured for one-directional propagation is give in Eqs. $(44)-(46)$, and for reference that set is reproduced here:

$$
\begin{aligned}
& \frac{d I(\zeta)}{d \zeta}=-\left[2(1-f) U_{e}(\zeta)+2 f \frac{I^{2}(\zeta)+I^{* 2}(\zeta)}{U_{e}(\zeta)}\right. \\
& -\frac{2\left(y+z_{0}\right) U_{e}(\zeta)}{z_{0}} \\
& \times \int_{-\infty}^{\infty} \frac{1}{1+(y-U)^{2}+U_{e}(\zeta)} \\
& \left.\times D_{0}(U, \zeta) d U\right] \text {, } \\
& \frac{d I^{*}(\zeta)}{d \zeta}=-\left[\delta\left(y+z_{0}\right) \frac{I^{2}(\zeta)+I^{* 2}(\xi)-U_{e}^{2}(\zeta)}{U_{e}(\xi)}\right. \\
& -\frac{2\left(y+z_{0}\right) U_{e}(\xi)}{z_{0}} \\
& \times \int_{-\infty}^{\infty} \frac{y-U}{1+(y-U)^{2}+U_{e}(\zeta)} \\
& \left.\times D_{0}(U, \zeta) d U\right] \\
& \frac{d U_{e}(\zeta)}{d \zeta}=-2\left[2 f I(\zeta)+\delta\left(y+z_{0}\right) I^{*}(\zeta)\right] .
\end{aligned}
$$

The limit of large $z_{0}$ corresponds to the derivative approximation, and in this limit the simplified form of Eqs. (A1) $-(\mathrm{A} 3)$ is

$$
\begin{aligned}
& \frac{d I(\zeta)}{d \zeta}=-\left[2(1-f) U_{e}(\zeta)+2 f \frac{I^{2}(\zeta)+I^{* 2}(\zeta)}{U_{e}(\zeta)}\right. \\
& -2 U_{e}(\zeta) \int_{-\infty}^{\infty} \frac{1}{1+(y-U)^{2}+U_{e}(\zeta)} \\
& \left.\times D_{0}(U, \zeta) d U\right] \\
& \frac{d I^{*}(\zeta)}{d \zeta}=-\delta z_{0} \frac{I^{2}(\zeta)+I^{* 2}(\zeta)-U_{e}^{2}(\zeta)}{U_{e}(\zeta)},
\end{aligned}
$$




$$
\frac{d U_{e}(\zeta)}{d \zeta}=-2 \delta z_{0} I^{*}(\zeta)
$$

In order for the derivative of $U_{e}(\xi)$ to remain finite for large values of $z_{0}$, it follows from Eq. (A6) that $I^{*}(\zeta)$ must approach zero for all values of $\zeta$. In the same limit Eq. (A5) then yields $I(\zeta)=U_{e}(\zeta)$. Finally, with this substitution the limiting form of Eq. (A4) is

$$
\begin{array}{r}
\frac{d I(\zeta)}{d \zeta}=-2 I(\zeta)\left[1-\int_{-\infty}^{\infty} \frac{1}{1+(y-U)^{2}+I(\zeta)}\right. \\
\left.\quad \times D_{0}(U, \zeta) d U\right]
\end{array}
$$

Thus, in this approximation the intensity is governed by a single equation, and in special cases this equation can be solved analytically.

The simplest special case of Eq. (A7) relates to the limits of homogeneous line broadening and constant pumping. In these limits the inversion function $D_{0}(U, \zeta)$ can be replaced by a $\delta$ function in frequency times the thresholds parameter, and Eq. (A7) reduces to

$$
\frac{d I(\zeta)}{d \zeta}=-2 I(\zeta)\left(1-\frac{r}{1+y^{2}+I(\zeta)}\right)
$$

This equation can be arranged into the form

$$
2 d \zeta=\frac{1+y^{2}+I}{I\left(r-1-y^{2}-I\right)} d I .
$$

Integrating yields

$$
\begin{aligned}
& \frac{2\left(r-1-y^{2}\right)}{1+y^{2}}\left(\zeta_{2}-\zeta_{1}\right) \\
& \quad=\ln \left(\frac{I_{2}}{I_{1}}\right)-\frac{r}{1+y^{2}} \ln \left(\frac{r-1-y^{2}-I_{2}}{r-1-y^{2}-I_{1}}\right),
\end{aligned}
$$

where $\ln$ is the natural logarithm. This result can be regarded as an implicit equation for the intensity as a function of distance in the laser amplifier. For the limit of line-center operation $(y=0)$, Eq. (A10) reduces to Eq. (5) of Rigrod [4].

Another limit of special interest concerns inhomogeneously broadened laser amplifiers. Inhomogeneous lineshape functions can often be approximated by a Gaussian distribution such as

$$
D_{0}(U, \zeta)=r \exp \left(-\epsilon^{2} U^{2}\right) / \pi
$$

where the natural damping ratio $\epsilon=\Delta v_{h}(\ln 2)^{1 / 2} / \Delta v_{i}$ is a measure of the relative widths of the homogeneous and inhomogeneous broadening functions, and in the inhomogeneous limit $r$ is the threshold parameter. If $\epsilon(1+I)^{1 / 2}$ is much less than unity, Eq. (A7) can be written

$$
\begin{aligned}
\frac{d I(\zeta)}{d \zeta}=-2 I(\zeta)(1- & \frac{r \exp \left(-\epsilon^{2} y^{2}\right)}{\pi} \\
& \left.\times \int_{-\infty}^{\infty} \frac{1}{1+(y-U)^{2}+I(\zeta)} d U\right] \\
= & -2 I(\zeta)\left[1-\frac{r \exp \left(-\epsilon^{2} y^{2}\right)}{[1+I(\zeta)]^{1 / 2}}\right]
\end{aligned}
$$

This equation can be arranged into the form

$$
2 d \zeta=\frac{(1+I)^{1 / 2}}{I\left[r \exp \left(-\epsilon^{2} y^{2}\right)-(1+I)^{1 / 2}\right]} d I .
$$

Integrating yields

$$
\begin{aligned}
& 2\left(\frac{r^{\prime 2}-1}{r^{\prime}}\right)\left(\zeta_{2}-\zeta_{1}\right) \\
& =\ln \left[\frac{\left(1+I_{2}\right)^{1 / 2}-1}{\left(1+I_{2}\right)^{1 / 2}+1} \frac{\left(1+I_{1}\right)^{1 / 2}+1}{\left(1+I_{1}\right)^{1 / 2}-1}\right] \\
& \quad+\frac{1}{r^{\prime}} \ln \left[\frac{I_{2}}{I_{1}}\right]-2 r^{\prime} \ln \left(\frac{r^{\prime}-\left(1+I_{2}\right)^{1 / 2}}{r^{\prime}-\left(1+I_{1}\right)^{1 / 2}}\right),
\end{aligned}
$$

where the frequency-corrected threshold parameter $r^{\prime}=r \exp \left(-\epsilon^{2} y^{2}\right)$ has been introduced. For the limit of line-center operation Eq. (A14) reduces to Eq. (11) of Rig$\operatorname{rod}[4]$.

It is also of interest to consider the stability of the steady-state loss-limited solutions to the approximate laser models, such as that described by Eq. (A8). The steady-state solution of Eq. (A8) is

$$
I=r-1-y^{2} \text {. }
$$

As in the text, the $\zeta$-dependent perturbation away from steady-state is defined by the equation

$$
I(\zeta)=I+I^{\prime}(\zeta)
$$

When Eq. (A16) is substituted into Eq. (A8) and the results linearized, one obtains the linear equation

$$
\frac{d I^{\prime}(\zeta)}{d \zeta}=-\frac{2\left(r-1-y^{2}\right)}{r} I^{\prime}(\zeta),
$$

where the steady-state intensity has been eliminated using Eq. (A15).

The derivative in Eq. (A17) can be eliminated by means of the exponential substitution

$$
I^{\prime}(\xi)=I^{\prime \prime} \exp (s \xi)
$$

Thus the oscillation frequency is given by the negative real number

$$
s=-\frac{2\left(r-1-y^{2}\right)}{r},
$$

and one finds that the steady-state solution of the approximate amplifier equation is always stable against small perturbations. For line-center tuning $(y=0)$ the frequency is 


$$
s=-\frac{2(r-1)}{r}
$$

and this result is the same as the real root $s_{1}$ of the more exact model as given in Eq. (105).

Approximate analytical formulas can also be obtained for the two-directional amplifiers governed by Eqs. (62)-(66), but for brevity such reductions are omitted here. Most studies of two-directional amplifiers have been somewhat restricted in generality and have had the primary purpose of calculating the output of twodirectional laser oscillators. Using both the derivative approximation and a low-gain-per-pass approximation, the two-directional model given here reduces to one which has been solved previously for homogeneously and inhomogeneously broadened laser oscillators [5]. Partial solutions for the homogeneously broadened high-gain oscillator case were given by Scott [6-8] and more completely but omitting longitudinal spatial hole burning by Rigrod $[9,10]$ and others.
*Permanent address: Department of Electrical Engineering, Portland State University, P.O. Box 751, Portland, OR 97207-0751.

[1] L. W. Casperson, preceding paper, Phys. Rev. A 44, 3291 (1991).

[2] CRC Standard Mathematical Tables, 27th ed., edited by W. H. Beyer (CRC, Boca Raton, FL, 1985), p. 9.

[3] L. W. Casperson and A. Yariv, IEEE J. Quantum Electron. QE-8, 69 (1972).
[4] W. W. Rigrod, J. Appl. Phys. 34, 2602 (1963).

[5] L. W. Casperson, Appl. Opt. 19, 422 (1980).

[6] A. C. Scott, IEEE Trans. Electron Devices ED-11, 41 (1964).

[7] A. C. Scott, Proc. IEEE 52, 325 (1964).

[8] A. C. Scott, Solid State Electron. 8, 551 (1965).

[9] W. W. Rigrod, J. Appl. Phys. 36, 2487 (1965).

[10] W. W. Rigrod, IEEE J. Quantum Electron. QE-14, 377 (1978). 